\title{
Optimal apodizers for the vector vortex coronagraph with on-axis telescopes
}

\section{Alexis Carlotti, Dimitri Mawet, Laurent Pueyo}

Alexis Carlotti, Dimitri Mawet, Laurent Pueyo, "Optimal apodizers for the vector vortex coronagraph with on-axis telescopes," Proc. SPIE 8864, Techniques and Instrumentation for Detection of Exoplanets VI, 88641P (26 September 2013); doi: 10.1117/12.2024511

SPIE Event: SPIE Optical Engineering + Applications, 2013, San Diego, California, United States 


\title{
Optimal apodizers for the vector vortex coronagraph with on-axis telescopes
}

\author{
Alexis Carlotti ${ }^{a, b}$, Dimitri Mawet ${ }^{c}$, Laurent Pueyo $^{d}$ \\ ${ }^{a}$ Princeton University, MAE, Olden st., Princeton, NJ, USA; \\ ${ }^{b}$ Université Joseph-Fourier, IPAG, 414 rue de la piscine, France; \\ ${ }^{c}$ European Southern Observatory, Alonso de Cordova 3107, Vitacura, Santiago, Chile; \\ ${ }^{d}$ Space Telescope Science Institute, 3700 San Martin Drive, Baltimore, MD 21218, USA;
}

\begin{abstract}
Phase mask coronagraphs such as the vortex coronagraph are meant to be used with the circular clear aperture of off-axis telescopes, and their performance is greatly reduced when used with on-axis telescopes. A possible solution to that problem consists in apodizing the entrance pupil plane of the coronagraph. Apodizers can be computed by solving a quadratic optimization problem in which the transmission of the apodizer is maximized under the constraint that the intensity of the electric field in the Lyot plane is less than an extremum value that is chosen to be small enough to sufficiently attenuate the starlight in the subsequent image plane. The transmission of the apodizer and the throughput of the system depends on the characteristics of the aperture and of the Lyot stop. We present apodizers optimally designed for the apertures of the Palomar telescope, the VLT, the E-ELT and the AFTA telescope, and for vortex phase masks with a topological charge of 4 . Transmissions of 50 to $80 \%$ are found for mean contrast of $10^{-6}$ in the Lyot plane. The end throughput is between $16 \%$ and $55 \%$, and mean contrast in the image plane at $2.5 \lambda / D$ is between $3 \times 10^{-8}$ and $3 \times 10^{-9}$.
\end{abstract}

Keywords: Coronagraphy, High-Resolution Imaging, Exoplanet direct detection

\section{INTRODUCTION}

As detailed in Mawet et al (2012) ${ }^{1}$, only a few coronagraphs can achieve inner working angles as small as 1.5$2 \lambda / D$ with $10^{-10}$ contrast. Among them is the vortex coronagraph ${ }^{2}(\mathrm{VC})$. Like other phase mask coronagraphs such as the four-quadrant phase mask ${ }^{3}(4 \mathrm{QPM})$, the $\mathrm{VC}$ is supposed to be used with the circular clear aperture of an off-axis telescope. When used with such an aperture this coronagraph moves all of the incoming on-axis light outside the aperture, so that it can be entirely blocked with a Lyot stop.

The VC uses a focal mask that induces an $e^{i \theta l}$ phase shift, where $\theta$ is the azymutal angle in the image plane, and $l$ is the Pancharatnam topological charge of the mask. The charge must be an even integer greater than 0 for the on-axis light to be entirely rejected outside the pupil.

The off-axis light is only attenuated when the off-axis source gets too close to the star. The inner working angle of the VC depends on its topological charge. For the smallest charge, i.e., l=2, the IWA of the VC equals $1 \lambda / D$. It becomes $1.7 \lambda / D$ if $\mathrm{l}=4$. The topological charge of the $\mathrm{VC}$ also constraints its sensitivity to pointing errors, jitter, and to the finite stellar size (the off-axis transmission of the coronagraph goes as $\theta^{l}$ ).

While the very small IWA of the charge $2 \mathrm{VC}$ is attractive, it is believed that a charge $4 \mathrm{VC}$ is a better choice. In both cases the $\mathrm{VC}$ is capable of creating $10^{-10}$ contrast.

The VC suffers from an important limitation, however. The central obscuration of telescopes has a significant impact on the contrast. This weakness is also shared with the 4QPM. In practice the VC has been used at the Hale telescope of the Palomar observatory, but only an unobscured fraction of the aperture has been used. ${ }^{4}$ It has also been used more recently ${ }^{5}$ in the NACO instrument installed at the very-large telescope (VLT). In the L' band, the coronagraphic contrast was $10^{-4}$ at $4 \lambda / D$ and it was slightly above $10^{-6}$ beyond $10 \lambda / D$. In the

Further author information: (Send correspondence to A.C.)

A.C.: E-mail: alexis.carlotti@obs.ujf-grenoble.fr

Techniques and Instrumentation for Detection of Exoplanets VI, edited by Stuart Shaklan, Proc. of SPIE Vol. 8864, 88641P · C 2013 SPIE · CCC code: 0277-786X/13/\$18 · doi: 10.1117/12.2024511 


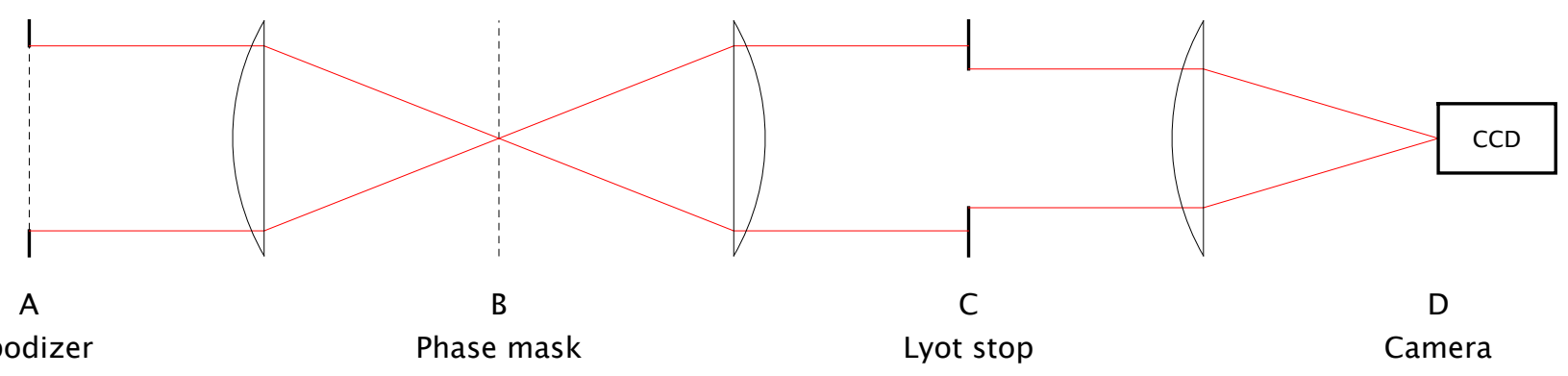

Figure 1: Layout of an AVC. The apodizer is located in place A, i.e., the first pupil plane. The VC is in plane B, one Fourier transform away from plane A. A second fourier transform brings light to the Lyot plane, or plane $\mathrm{C}$, where the Lyot stop is located. The final image plane is the location of the camera.

facilities of the high-contrast imaging laboratory (HCIT), and using a clear aperture, a $2.5 \times 10^{-8}$ contrast has been measured in a $10 \%$ band, while a monochromatic $4 \times 10^{-9}$ contrast has been measured at $785 \mathrm{~nm}$.

Apodizers can be used with phase mask coronagraphs to create high-contrast in spite of a central obscuration. A recent paper $^{6}$ has showed, using numerical simulations, that optimal apodizers associated with a 4QPM coronagraph at the VLT could deliver $10^{-9}$ contrast at $1 \lambda / D$ from the star. In an upcoming paper (Mawet et al., 2013, submitted), a similar proof of concept is presented for the VC. Fig.?? shows the basic layout of an apodized vortex coronagraph (AVC).

In this paper we present apodizers optimized for a charge $4 \mathrm{VC}$ and four telescope apertures: those of the Palomar telescope, the VLT, the european extremely large telescope (EELT), and of the astronomical focused telescope assets (AFTA). For each of these four apertures, we have computed various masks and identified the most optimal ones by varying the central obscuration of the associated Lyot stop.

In Sec.2 we recall the mathematical formalism that is the basis for these apodizers computations. Sec.3 presents the results obtained for the apertures of the three ground-based telescopes previously mentioned, and Sec.4 does the same for WFIRST-AFTA. Sec.5 draws a conclusion to the paper.

\section{COMPUTING OPTIMAL APODIZERS FOR THE VORTEX CORONAGRAPH}

The computation of optimal apodizers for the VC requires (a) an understanding of the mathematical formalism used to evaluate the electric field in the Lyot plane based on the electric field in the entrance pupil plane, (b) knowing the constraints that the Nyquist-Shannon sampling theorem sets on the actual computation of this electric field, and (c) a clear description of the quadratic optimization problem that must be solved.

\subsection{Mathematical formalism}

This formalism has first been introduced in a previous paper ${ }^{6}$, and it does not appear necessary to recall it here in its entire form. If the apodized aperture has a transmission $A(x, y)$, the electric field in the Lyot plane is proportional to the convolution of $A(x, y)$ and $F(x, y)$ :

$$
P(\tilde{x}, \tilde{y})=e^{4 i \pi F / \lambda} A(x, y) * F(x, y),
$$

where $F(x, y)$ is the Fourier transform of the focal plane mask $M(u, v)$ :

$$
F(x, y)=\iint_{-\infty}^{\infty} M(u, v) e^{2 i \pi(x u+y v)} d u d v .
$$

If a closed form for the function $F(x, y)$ can be derived, then Eq.2 can be used to compute the electric field in the Lyot plane without explicitly computing the electric field in the intermediate image plane. This is the most interesting feature of this formalism: it is not sensitive to errors due to the discretized nature of the mask. 
The calculation of $F(x, y)$ depends on the chosen phase mask $M(u, v)$. For the $\mathrm{VC}$ it is more convenient to write the expression of $F(x, y)$ as a double integral with respect to $\rho$ and $\theta$, where $\rho$ and $\theta$ are the radial distance and the azymutal angle in the $(u, v)$ plane. The function $M(\rho, \theta)$ does not depend on $\rho$, and equals $e^{i \theta l}$, where $l$ is the topological charge. This property makes it possible to proceed first with the integration with respect to $\rho$ :

$$
\begin{aligned}
& F(x, y)=\int_{0}^{2 \pi} \int_{0}^{L} \rho M(\theta) e^{2 i \pi \rho(x \cos (\theta)+y \sin (\theta))} d \rho d \theta=\int_{0}^{2 \pi} M(\theta) \Psi(x \rho \cos (\theta)+y \rho \sin (\theta)) d \theta \\
& \text { where } \Psi(r)=\int_{0}^{L} \rho e^{2 i \pi \rho r} d \rho=\frac{(1-2 i \pi L r) e^{2 i \pi L r}-1}{4 \pi^{2} r^{2}} .
\end{aligned}
$$

Note that $L$ represents the radius of the image plane mask (in units of $\lambda / D$ ). While it is usually assumed that the mask is infinitely large, it is necessary to specify its size in order to derive the closed form expression for $\Psi(r)$ given in Eq.3. The expression of $F(x, y)$ has previously been derived for a charge 2 VC:

$$
\begin{aligned}
& F(x, y)=\frac{e^{2 i \phi(x, y)}}{\pi r(x, y)^{2}}\left[-1+J_{0}(2 \pi L r(x, y))+\pi L r(x, y) J_{1}(2 \pi L r(x, y))\right] \\
& \text { where } r(x, y)=\sqrt{x^{2}+y^{2}}, \text { and } \phi(x, y)=\tan ^{-1}(y / x) .
\end{aligned}
$$

In the case of a topological charge $l=4$, the integration over $\theta$ gives the following result:

$$
F(x, y)=\frac{e^{4 i \phi(x, y)}}{\pi r(x, y)^{2}} \times\left[2+4 J_{0}(2 \pi L r(x, y))+\left(\pi L r(x, y)-\frac{6}{\pi L r(x, y)}\right) J_{1}(2 \pi L r(x, y))\right] .
$$

It is possible to derive a general expression for any even, positive integer value $l$ by rewriting Eq.3, and integrating first over $\theta$ :

$$
\begin{aligned}
F(x, y) & =\int_{0}^{2 \pi} \int_{0}^{L} \rho e^{i l \theta} e^{2 i \pi(x \rho \cos (\theta)+y \rho \sin (\theta))} d \rho d \theta \\
& =\int_{0}^{2 \pi} \int_{0}^{L} \rho e^{i l \theta} e^{2 i \pi r \rho \cos (\theta-\phi)} d \rho d \theta \\
& =\int_{0}^{L} \rho e^{i l \phi}\left(\int_{0}^{2 \pi} e^{i l \tilde{\theta}} e^{2 i \pi r \rho \cos \tilde{\theta}} d \tilde{\theta}\right) d \rho,
\end{aligned}
$$

where we have first written $x=r \cos \phi, y=r \cos \phi$, and then proceeded with the change of variables $\tilde{\theta}=\theta-\phi$. We recognize here an expression which is closely related to the definition of the Bessel function of the $l$ th order. The expression of $F(x, y)$ becomes:

$$
F(x, y)=(-1)^{l} e^{i l \phi} \int_{0}^{L} 2 \pi J_{l}(2 \pi r \rho) \rho d \rho,
$$

and the final expression for $F(x, y)$ is obtained after integrating over $\rho$ :

$$
F(x, y)=e^{i l \phi} \frac{2 \pi L^{2}(\pi L r)^{l}}{(2+l) \Gamma(l+1)}{ }_{1} F_{2}\left(1+l / 2 ; 2+l / 2,1+l ;-(\pi L r)^{2}\right),
$$

where $F$ is the generalized hypergeometric function. Numerical applications show that Eq. 8 is in accordance with Eq.4, and 5, as well as with more traditional approaches where the intermediate image plane is explicitly computed.

\subsection{Discretization considerations}

We discretize plane A using $\mathrm{N}$ points along both axes, and we do the same in plane $\mathrm{C}$ (the Lyot plane) with $\mathrm{M}$ points. 
Both the 4QPM and the VC, used with a circular clear aperture, produce ripples in the Lyot plane. If the mask extends up to $\mathrm{L} \lambda / D, 2 \mathrm{~L}$ ripples appear in the Lyot plane. The Nyquist-Shannon sampling theorem states that each period should be sampled with two points, thus $M=2 \times L$. Observing up to $32 \lambda / D$ implies that M equals at least 64 . This is the case for all the apodizers presented in this paper.

$\mathrm{N}$ should be as high as possible. Its value depends on $\mathrm{L}$, and on the available RAM. About 16-32GB are required for an apodizer discretized over 512 by 512 points. Note that the 4-fold symmetry of most telescope apertures can be used to greatly relax the constraint that RAM sets on N: twice as many points can be used if the mask is optimized over one quadrant of the pupil plane.

We transcribe this problem using $\mathrm{AMPL}^{7}$, and solve it with either one of two solvers: $\mathrm{LOQO}^{8}$ or Gurobi. ${ }^{9}$ LOQO has the advantage of handling some non-linear problems that Gurobi cannot solve. Gurobi on the other end accepts parallel computing.

The complexity of the computation goes as $N^{2} M^{2}$. Note that the function $\mathrm{F}(\mathrm{x}, \mathrm{y})$ cannot be separated into a product of a function of $\mathrm{x}$ and a function of $\mathrm{y}$. Otherwise the discrete computation of Eq.1 could have been written as two nested sums, making the complexity $N^{2} M+N M^{2}$.

\subsection{Quadratic optimization problem}

The apodizer is discretized over $\mathrm{N}$ points along each axis of the pupil plane. Since we assume that the aperture diameter equals 1 , the distance between nearest neighbors is $\Delta x=\Delta y=\frac{1}{N}$.

The problem consists in maximizing the total transmission of the mask $\sum_{i=1}^{N} \sum_{j=1}^{N} A\left(x_{i}, y_{j}\right) \Delta x \Delta y$ under the following constraints set on the extremum values of the transmission of the apodizer, and of the amplitude of the electric field $P\left(\tilde{x}_{k}, \tilde{y}_{l}\right)$ in the Lyot plane:

$$
\begin{array}{r}
0<A\left(x_{i}, y_{j}\right)<1, \text { with }\left\{x_{i}, y_{j}\right\} \in \Delta_{A} \\
\left|P\left(\tilde{x}_{k}, \tilde{y}_{l}\right)\right|^{2} \leq 10^{-c}, \text { with }\left\{\tilde{x}_{k}, \tilde{y}_{l}\right\} \in \Delta_{C},
\end{array}
$$

where $\Delta_{A}$ is the region defined by the telescope's aperture, $\Delta_{C}$ is the region defined by the Lyot stop, and $c$ measures the attenuation of the intensity in the reimaged pupil plane in a logarithmic scale.

To some extent this problem can be compared to the optimization of $2 \mathrm{D}$ shaped pupils ${ }^{10}$. Because of the finite angular extent of the vortex phase mask, the results of such optimization problems are here also binary apodizers. The first shaped pupils have originally been optimized in one dimension and considered for the terrestrial planet finder project. ${ }^{11,12}$

\section{OPTIMAL APODIZERS FOR THREE MAJOR GROUND-BASED TELESCOPES}

We present in this section the optimal apodizers that we have computed for the apertures of the 200" Hale telescope at the Palomar observatory (also referred to as the Palomar telescope), one of the four $8.2 \mathrm{~m}$ unit telescopes at the VLT, and the $39 \mathrm{~m}$ EELT. Three reasons explain the choice of these apertures:

- they offer an interesting diversity in term of obscuration size, spider thickness and spider configuration.

- the VC has been used at the Palomar telescope and the VLT.

- the VC could potentially be used at the EELT, for instance as part of the EPICS instrument.

\subsection{Apertures of the Palomar telescope, the VLT, and the EELT}

It is necessary to describe the properties of the apertures for which these apodizers are computed. This is especially important since some parameters - like the spider thickness - appear to have an important effect on the solution.

Tab.1 summarizes the information related to the apertures of these three major ground-based telescope, which are also displayed in Fig.2a, 2b, and 2c. 


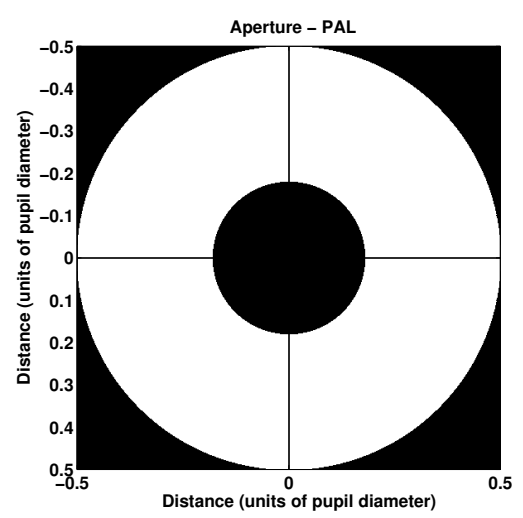

(a)

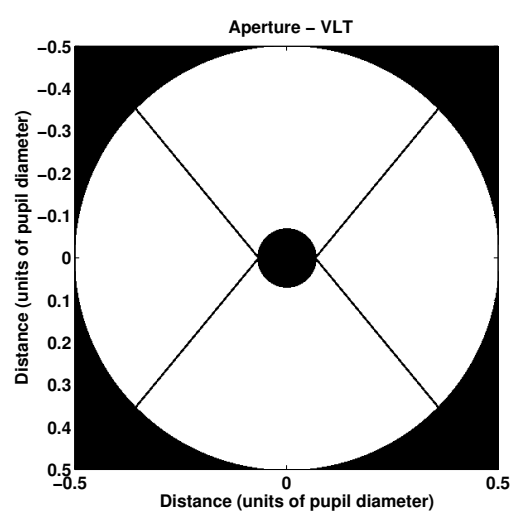

(b)

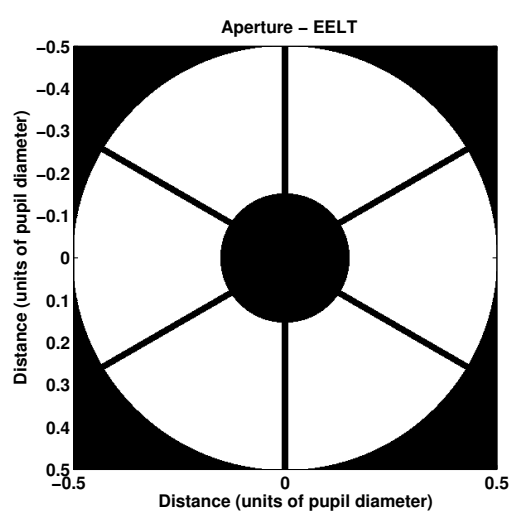

(c)

Figure 2: Apertures of (a) the Palomar telescope, (b) the very large telescope, and (c) the European extremely large telescope. Axes are in units of pupil diameter.

The apertures used in these computations are close to their original, but they are not identical. Our goal here is not to present the definitive version of the masks that could be used in a real AVC, but to get a fair estimate of their performance.

We do not simulate the gaps between the segments of the EELT, nor do we simulate the polygonal outer and inner edges of the aperture. Taking these details into account will require a computer with more RAM (possibly up to 512GB). A more careful analysis should be the subject of further publications.

\subsection{Optimal apodizers and Lyot stops}

As described in the case of the $4 \mathrm{QPM}^{6}$, the choice of the Lyot stop has a large influence on the maximum throughput of the coronagraph.

Our Lyot stops are modeled after the aperture to which they are associated: the outer edge is undersized, the inner edge is oversized, and so are the spiders. We have chosen to consider masks with radius of $\mathrm{L}=32 \lambda / D$, and the outer edge of the Lyot stop must be at least $4 \%$ smaller than the aperture to be free from the diffraction effects that the finite size of the vortex phase mask create (this roughly corresponds to $1 / \mathrm{L}$ ).

Oversizing the spiders is necessary too, although it only has a significant influence when the apertures have spiders with a large thickness. We have considered several different oversize factors, and we settled for Lyot stop spiders twice as large as those of the pupil.

The most influential parameter is the central obscuration of the Lyot stop. Too small, and the diffraction effects due to the central obscuration cannot be compensated. Too large and the throughput of the AVC becomes smaller than what it could be.

An analytical method presented in the aforementioned upcoming paper by Mawet et al. makes it possible to obtain a first estimate of the Lyot stop obscuration. We thus start from this estimate and change its value to look for the combination with the highest throughput.

Tab.2 summarizes the parameters and performance of the AVC for these three apertures.

\begin{tabular}{|c|c|c|c|c|}
\hline Telescope & Diameter $(\mathrm{m})$ & Obscuration (\%) & spider configuration & spider thickness (\%) \\
\hline \hline Palomar (Hale) & 5.1 & 36 & orthogonal & 0.25 \\
\hline VLT & 8.2 & 14 & 4-fold, non-orthogonal & 0.5 \\
\hline EELT & 39.3 & 30 & hexagonal & 1.5 \\
\hline
\end{tabular}

Table 1: Main properties of the apertures of the Palomar telescope, very-large telescope, and European extremelylarge telescope. 
The transmission of the apodizers are showed in Fig3a, 3b, and 3c. The electric field for an on-axis source a star - in the Lyot plane is displayed in a log scale in Fig.3d, 3e, and 3f. Finally, the electric field for an off-axis source - a planet - in the Lyot plane is presented in Fig.3g, 3h, and 3i.

It is particularly interesting to note that the throughput of the AVC optimized for the Palomar telescope is higher than the one obtained for the EELT, despite the fact that the central obscuration of the former is larger than the latter. The only reasons that explains this higher throughput are the much thinner spiders assumed for the Palomar telescope ( $0.25 \%$ of the pupil diameter, instead $1.5 \%$ for the EELT), and the larger number of spiders in the aperture of the EELT.

The off-axis transmissions are showed in Fig.4d, 4e, and 4f. These transmission are normalized to the maximum throughput for each AVC, which is reached at 20-25 $/ D$. The transmission does not increase monotonously: it stalls at about $1.5 \lambda / D$ and can even decrease slightly before increasing again. This has an important influence on the IWA of the AVC. Note that the original charge $4 \mathrm{VC}$ (with a clear aperture, and no apodization) has a $1.7 \lambda / D$ IWA.

The IWA is defined here as the angular distance at which $50 \%$ of the maximum throughput is already transmitted. This definition was proposed by Guyon. ${ }^{13}$

The IWA increases with the central obscuration and is not as much influenced by the spider thickness as the throughput is. It is $2.2 \lambda / D$ for VLT (which has the smallest obscuration), and $3.2 \lambda / D$ for the Palomar telescope (which has the largest obscuration).

Because of the way the quadratic optimization problem is solved, the contrast is computed relative to the apodizer transmission. This explains why the contrast measured in the image plane goes with the throughput.

The contrast obtained for the VLT and the Palomar telescope may actually be too low. These observatories are currently not equipped with a wavefront control system capable of ensuring that these contrasts could be matched. The constraint on the contrast could be relaxed so as to increase the throughput of the AVC.

\section{OPTIMAL APODIZERS FOR WFIRST-AFTA}

A coronagraph in WFIRST-AFTA is a tremendous opportunity for the direct imaging of exoplanets. In a recent report $^{14}$, the specifications of this coronagraph have been detailed: a $10^{-9}$ contrast must be obtained a 0.1 arcsec, which is $3 \lambda / D$ at $400 \mathrm{~nm}$.

These specifications are all the more challenging that the aperture of AFTA has a 29\% central obscuration, and six $1.7 \%$ thick spiders attached to the secondary with three large brackets that make the effective central obscuration 34\%. In our optimizations we have chosen to cover these brackets. Note that the aperture of AFTA is potentially subject to change.

As already noted by comparing the cases of EELT and the Palomar telescope, the spiders thickness plays an important role in the throughput. We have chosen to investigate this problem by considering four different thicknesses for the spiders of AFTA: $1.75 \%$ (the nominal value), $1 \%, 0.5 \%$, and $0.25 \%$ (the thickness of the spiders at the Palomar telescope).

The results of the optimizations are summarized in Tab.3. The impact of the spiders thickness becomes even more apparent.

\begin{tabular}{|c|c|c|c|c|c|c|}
\hline Telescope & $T_{\text {obs }}$ & $L S_{\text {obs }}$ & $A_{T}$ & Throughput & IWA & Contrast (IWA) \\
\hline \hline Palomar (Hale) & $36 \%$ & $70 \%$ & $76 \%$ & $31 \%$ & $3.2 \lambda / D$ & $8 \times 10^{-9}$ \\
\hline VLT & $14 \%$ & $42 \%$ & $79 \%$ & $55 \%$ & $2.2 \lambda / D$ & $4 \times 10^{-9}$ \\
\hline EELT & $30 \%$ & $58 \%$ & $49 \%$ & $16 \%$ & $2.9 \lambda / D$ & $3 \times 10^{-8}$ \\
\hline
\end{tabular}

Table 2: Main parameters for the AVC optimized for the Palomar telescope, the VLT and the EELT. $T_{\text {obs }}$ refers to the central obscuration of the telescope. $L S_{o b s}$ is the central obscuration of the Lyot stop. $A_{T}$ is the apodizer transmission. 


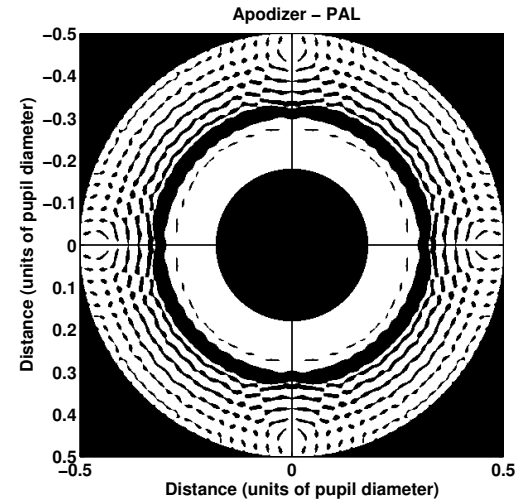

(a)

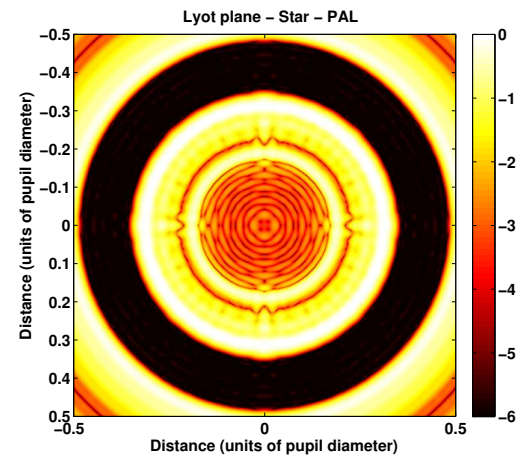

(d)

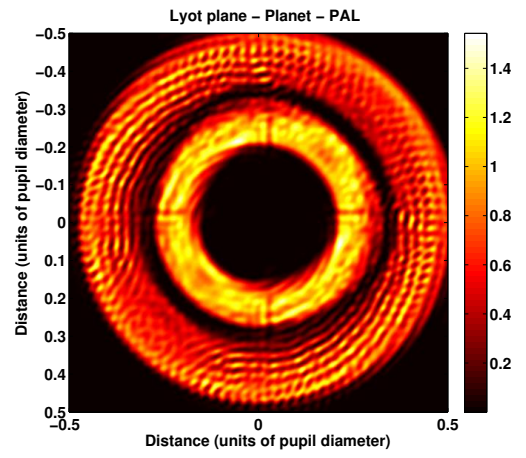

(g)

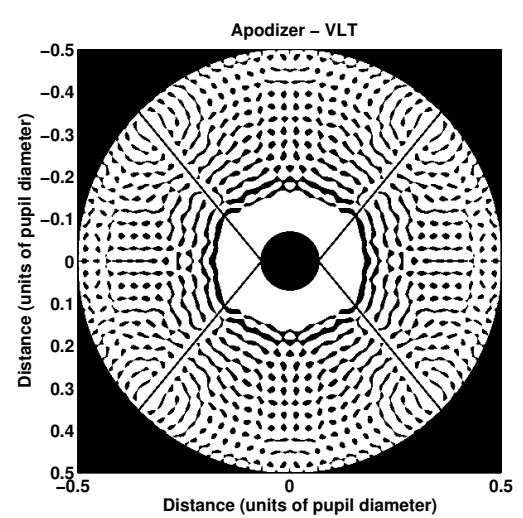

(b)

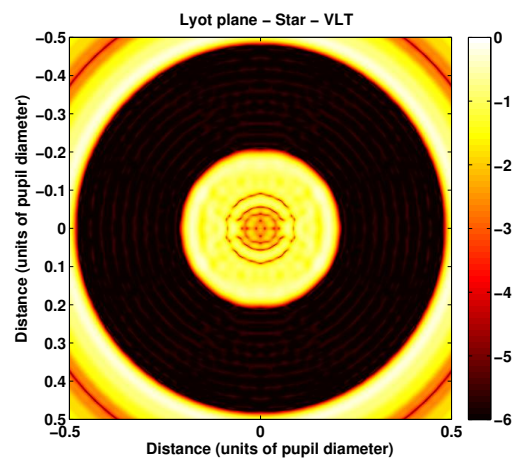

(e)

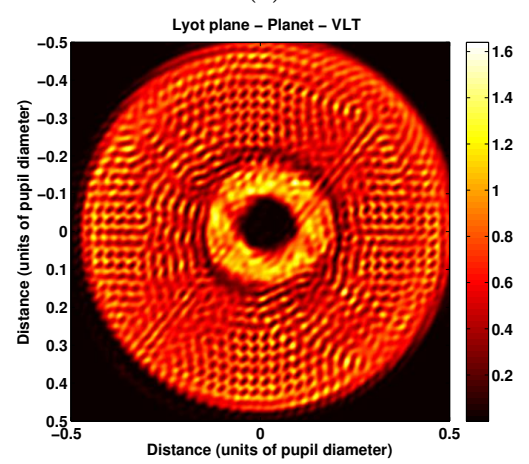

(h)

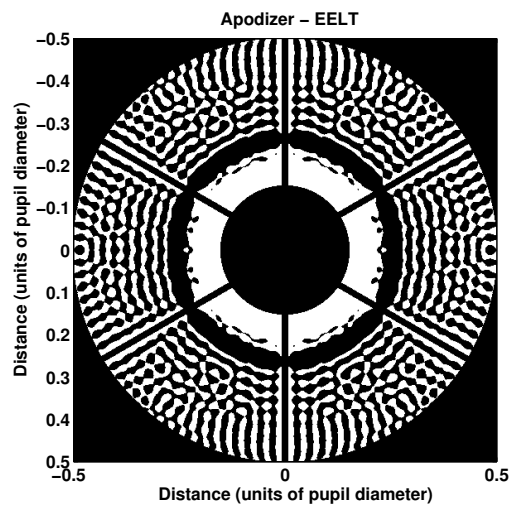

(c)

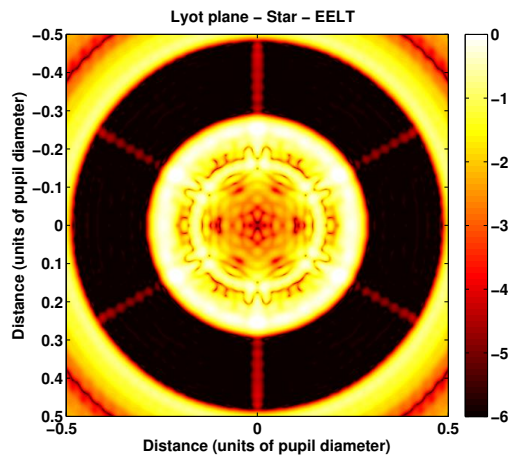

(f)

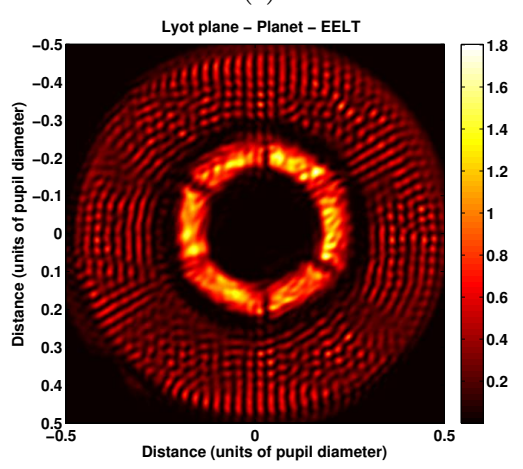

(i)

Figure 3: First row: optimal apodizers for (a) the Palomar telescope, (b) the very large telescope, and (c) the European extremely large telescope. Axes are in units of pupil diameter. Second row: energy in the Lyot plane for an on-axis source (log scale). Third row: energy in the Lyot plane for an off-axis source (linear scale). The off-axis source is located at $25 \lambda / D$ along the diagonal.

\begin{tabular}{|c|c|c|c|c|c|c|}
\hline$S_{t}$ & $T_{\text {obs }}$ & $L S_{\text {obs }}$ & $A_{T}$ & Throughput & IWA & Contrast (IWA) \\
\hline \hline $0.25 \%$ & $34 \%$ & $70 \%$ & $79 \%$ & $32 \%$ & $2.5 \lambda / D$ & $8 \times 10^{-10}$ \\
\hline $0.5 \%$ & $34 \%$ & $70 \%$ & $76 \%$ & $29 \%$ & $2.9 \lambda / D$ & $3 \times 10^{-9}$ \\
\hline $1.0 \%$ & $34 \%$ & $70 \%$ & $67 \%$ & $22 \%$ & $3.1 \lambda / D$ & $5 \times 10^{-9}$ \\
\hline $1.75 \%$ & $34 \%$ & $70 \%$ & $53 \%$ & $12 \%$ & $3.2 \lambda / D$ & $2 \times 10^{-8}$ \\
\hline
\end{tabular}

Table 3: Main parameters for the AVC optimized for WFIRST-AFTA with 4 different spiders thicknesses $(0.25 \%$, $0.5 \%, 1 \%$, and $1.75 \%$ of the pupil diameter). $S_{t}$ is the spider thickness. $T_{o b s}$ refers to the central obscuration of the telescope. $L S_{\text {obs }}$ is the central obscuration of the Lyot stop. $A_{T}$ is the apodizer transmission. 


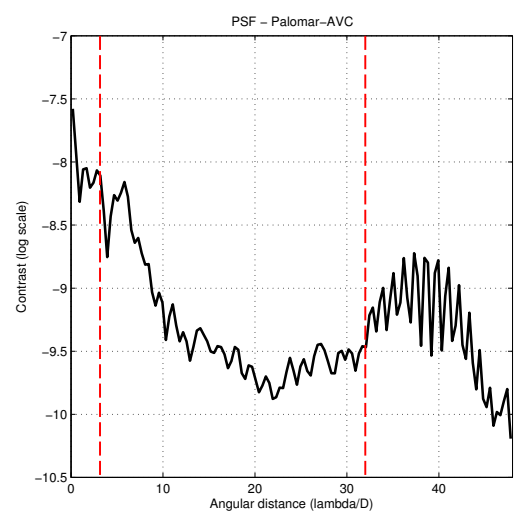

(a)

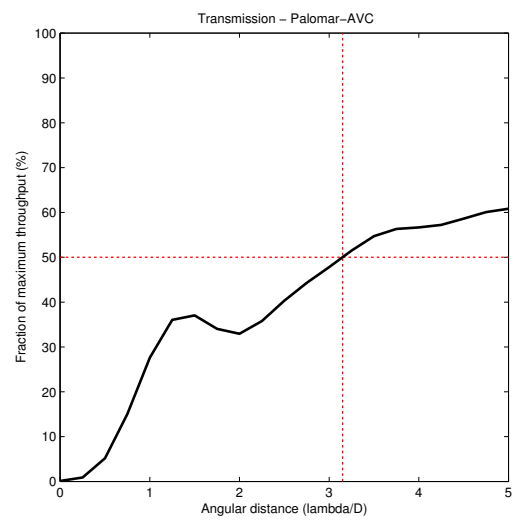

(d)

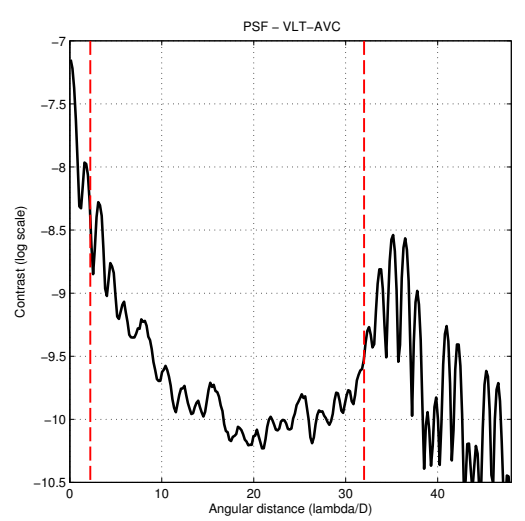

(b)

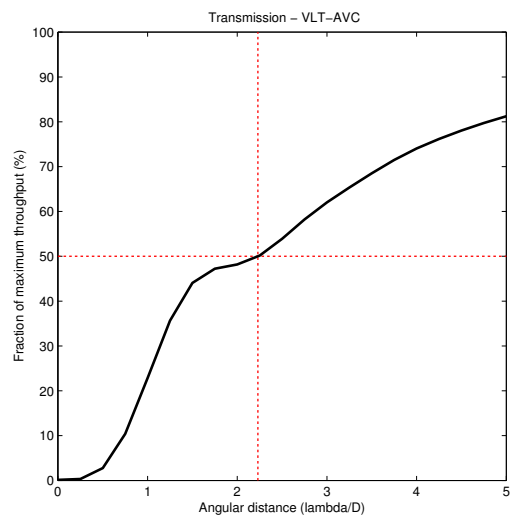

(e)

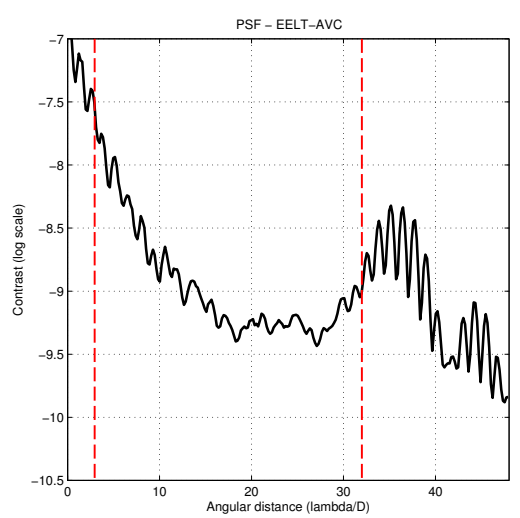

(c)

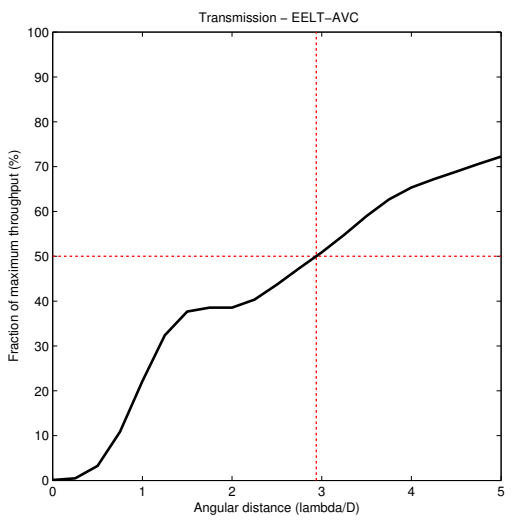

(f)

Figure 4: First row: azymutal averages of the PSF for: (a) the Palomar telescope, (b) the very large telescope, and (c) the European extremely large telescope. Second row: normalized transmission. The red dashed lines indicate the IWA of each AVC. Axes are in units of $\lambda / D$.

The effect of the spider thickness on the throughput is roughly linear. Each extra percent on the spider thickness decreases the throughput by $\approx 14 \%$. The two thinnest spiders $(0.25 \%$ and $0.5 \%)$ have a small impact on the throughput (32\% and 29\%). The throughput becomes $22 \%$ for $1 \%$ thick spiders, and $12 \%$ for the $1.75 \%$ thick spiders.

Here too - and for the same reason - the contrast is affected by the throughput. Its highest value (for the $1.75 \%$ thick spiders) is $5 \times 10^{-8}$ at $2.5 \lambda / D$ from the star. An azymutal average of the contrast is displayed in Fig.6a for the four spider thicknesses. The off-axis transmission is showed in Fig.6b. It is not the absolute transmission, but the relative transmission, normalized with respect to the maximum transmission of each AVC (which roughly corresponds to a $20-25 \lambda / D$ angular distance).

The IWA is also indicated in Fig.6b. Contrary to the classical VC, the off-axis transmission is not monotonous with the AVC. The throughput increases and reaches a local maximum of $35-45 \%$ of the maximum throughput at $1.3-1.5 \lambda / D$ from the star. It then decreases slightly before increasing again.

While in the previous section it has been indicated that the IWA was mostly influenced by the pupil central obscuration, this parameter does not change here, and the IWA increases with the spider thickness, from $2.5 \lambda / D$ to $3.2 \lambda / D$. Note that the IWA of a classical $\mathrm{VC}$ is $1.7 \lambda / D$.

\section{DISCUSSION AND CONCLUSION}

In this paper we have outlined the performance of apodized vortex coronagraphs for four telescopes. Three of them are ground-based telescopes (the 200" telescope at the Palomar observatory, the VLT, and the EELT). 


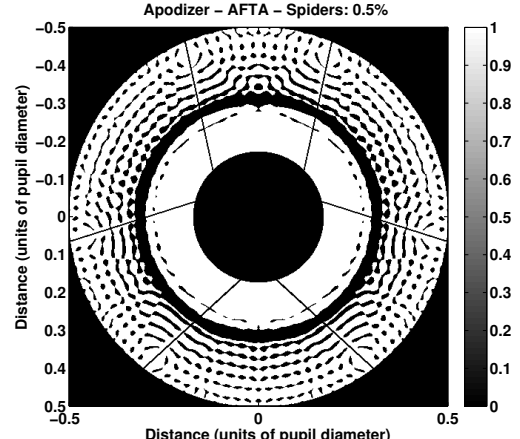

(a)

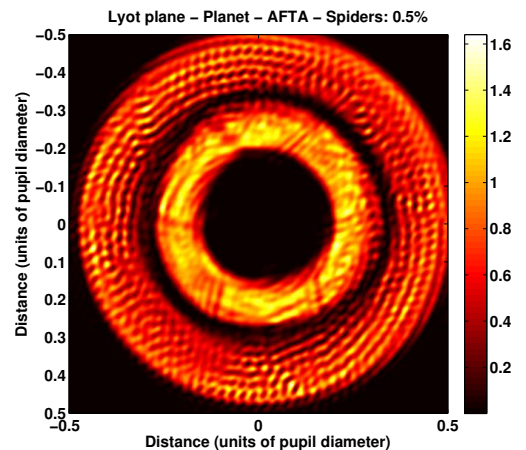

(d)

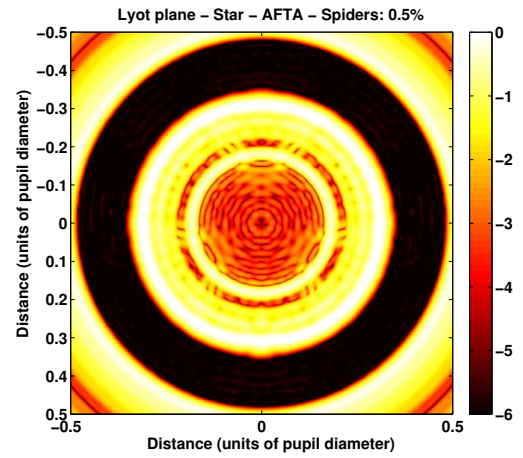

(g)

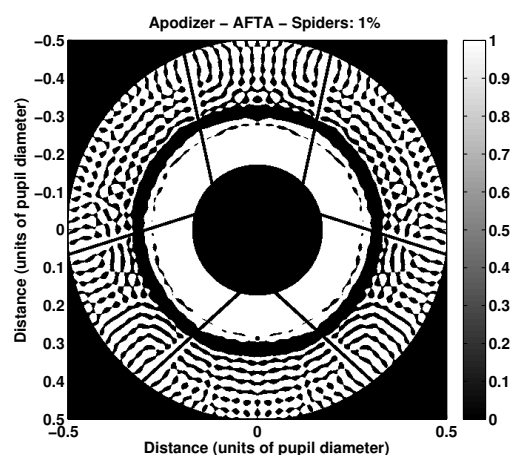

(b)

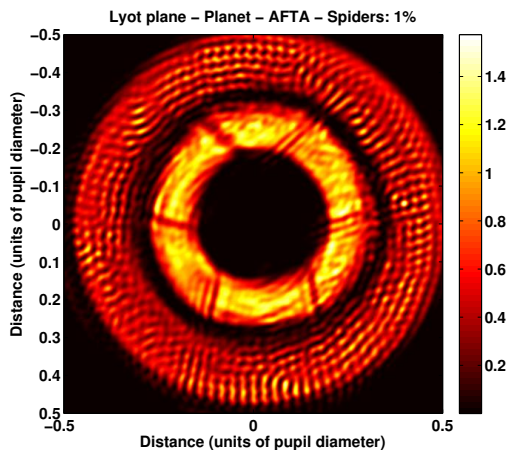

(e)

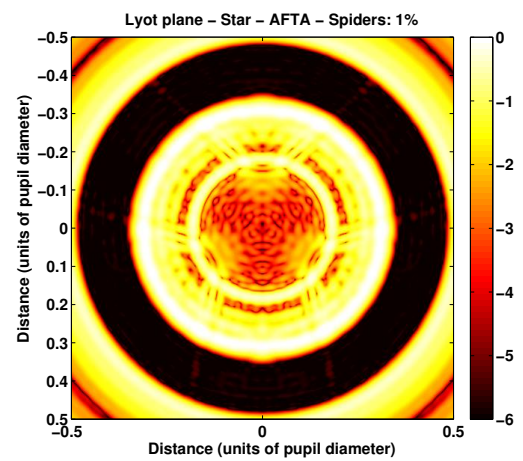

(h)

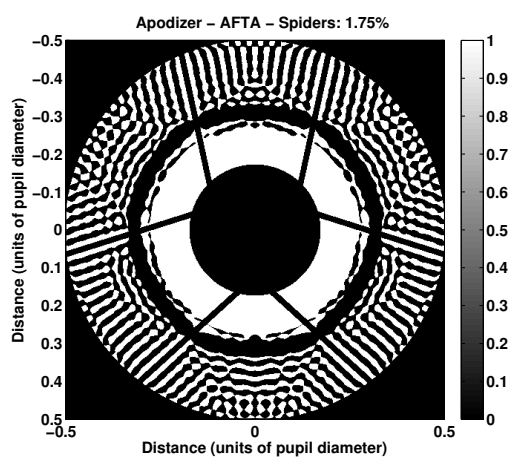

(c)

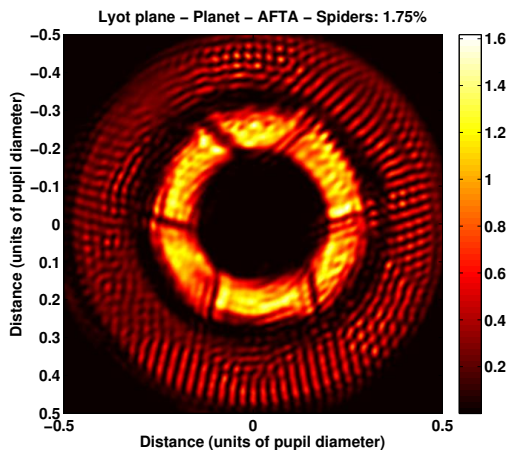

(f)

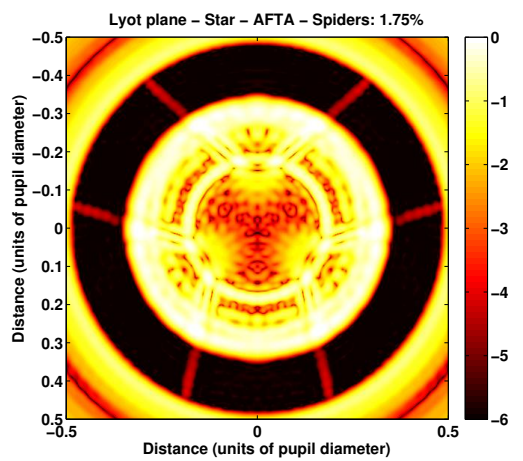

(i)

Figure 5: First row: optimal apodizers for WFIRST-AFTA with (a) $0.5 \%$ thick spiders, (b) $1 \%$ thick spiders, and (c) $1.75 \%$ thick spiders. Axes are in units of pupil diameter. Second row: energy in the Lyot plane for an on-axis source (log scale). Third row: energy in the Lyot plane for an off-axis source (linear scale). The off-axis source is located at $25 \lambda / D$ along the diagonal. 


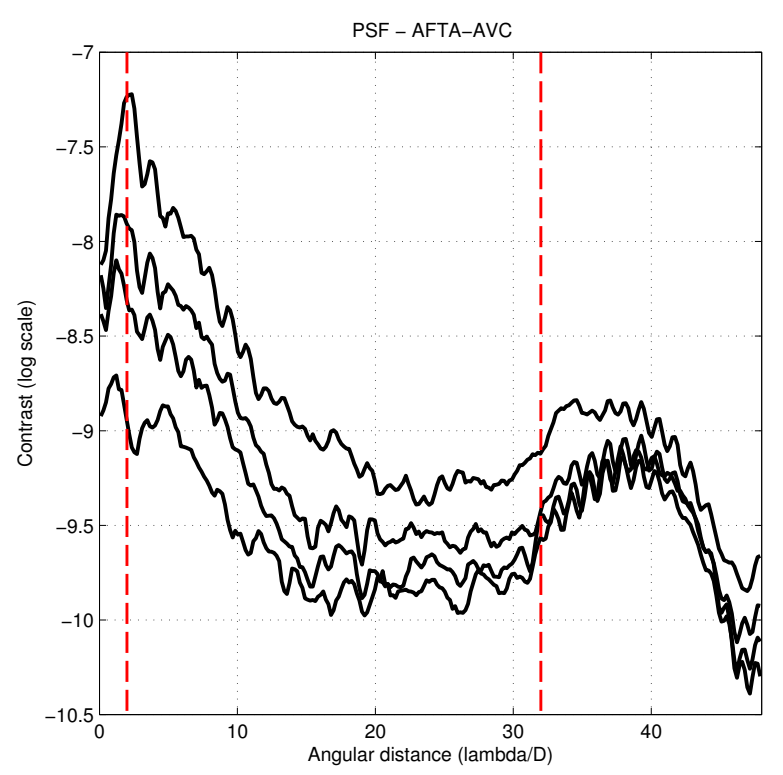

(a)

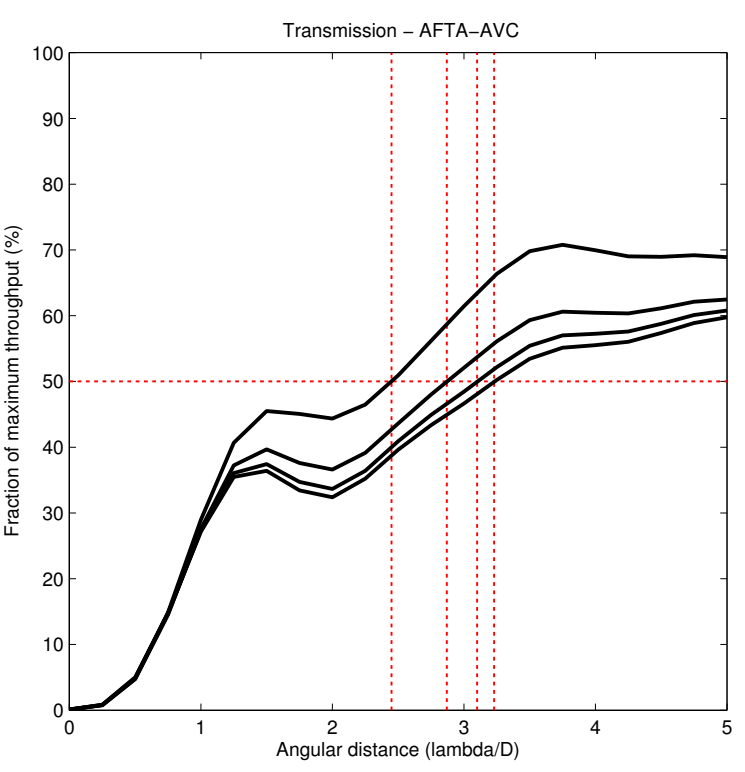

(b)

Figure 6: (a): Azymutal average of the contrast in plane D. From top to bottom the four solid black lines represent the contrast of the AVC optimized for the AFTA apertures with $1.75 \%, 1 \%, 0.5 \%$ and $0.25 \%$ thick spiders. The left red dashed line is located at $2 \lambda / D$, while the right red dashed line is located at $32 \lambda / D$, and indicates the radius of the phase mask. (b): Off-axis normalized transmissions (100\% corresponds to the maximum throughput of each coronagraph). The horizontal dashed red lines indicate the IWA of the coronagraph. The transmission curves correspond from top to bottom to the $0.25 \%, 0.5 \%, 1 \%$ and $1.75 \%$ thick spiders.

\section{The fourth one is WFIRST-AFTA.}

The vortex coronagraph for which we have optimized apodizers all have a Pancharatnam topological charge 4 , making them fairly insensitive to jitter and to the finite stellar size. In addition, we have limited the radius of the vortex phase mask in the image plane to $32 \lambda / D$.

The central obscuration of the aperture, and the thickness of its spiders drive the throughput of the AVC. Fairly high throughputs (30-50\%) are obtained for apertures with thin spiders (0.25-0.5\% of the pupil diameter). Thicker spiders drive the throughput down: $1.5 \%$ thick spiders in the aperture of the EELT make the throughput $16 \%$, and $1.75 \%$ thick spiders in the aperture of WFIRST-AFTA make the throughput $12 \%$.

Contrary to what can be observed with the VC, the off-axis transmission of the AVC is not monotonous. It stalls at around $1.5 \lambda / D$, which shifts the IWA from $1.7 \lambda / D$ IWA (for a charge $4 \mathrm{VC}$ ), to $2.5-3.2 \lambda / D$ (following the IWA definition proposed by Guyon), and it is influenced both by the pupil central obscuration and by the thickness of the spiders.

The sensitivity to the spider thickness is an issue that could potentially be bypassed by using a pupil mapping technique such as ACAD. ${ }^{15}$ The same technique could also relax the constraints that the central obscuration sets on the throughput of the AVC.

One of the attractive aspects of the combinations of shaped pupils and vortex coronagraph presented in this paper is their manufacturability. Several generations of vortex phase masks have been manufactured and tested. They have demonstrated $10^{-9}$ contrast at $2 \lambda / D$ in the HCIT.

Shaped pupils are also very well known components - they can be manufactured using the same process used for the microdots apodizers of the GPI and the SPHERE projects. As they use a glass substrate, internal reflections limit the contrast to a few $10^{-8}$ contrast as demonstrated in the laboratory ${ }^{16}$ during the development of the SPICA coronagraphic instrument. Alternative manufacturing techniques that do not require a transmissive substrate are currently investigated. 
It should be noted that the exact characteristics of the AFTA aperture are currently not know, and the nominal value of $1.7 \%$ for its spiders thickness may be pessimistic. It is also possible that the thickness of the spiders change in the future.

The same can be said of the EELT: it is not clear that the aperture that we have considered is the one that will be used. We intend to resume this work with a more careful study in which the telescope segmentation will be taken into account.

\section{REFERENCES}

[1] Mawet, D., Pueyo, L., Lawson, P., Mugnier, L., Traub, W., Boccaletti, A., Trauger, J. T., Gladysz, S., Serabyn, E., Milli, J., Belikov, R., Kasper, M., Baudoz, P., Macintosh, B., Marois, C., Oppenheimer, B., Barrett, H., Beuzit, J.-L., Devaney, N., Girard, J., Guyon, O., Krist, J., Mennesson, B., Mouillet, D., Murakami, N., Poyneer, L., Savransky, D., Vérinaud, C., and Wallace, J. K., "Review of small-angle coronagraphic techniques in the wake of ground-based second-generation adaptive optics systems," in [Society of Photo-Optical Instrumentation Engineers (SPIE) Conference Series], Society of Photo-Optical Instrumentation Engineers (SPIE) Conference Series 8442 (Sept. 2012).

[2] Mawet, D., Riaud, P., Absil, O., and Surdej, J., "Annular Groove Phase Mask Coronagraph," ApJ 633, 1191-1200 (Nov. 2005).

[3] Rouan, D., Riaud, P., Boccaletti, A., Clénet, Y., and Labeyrie, A., "The Four-Quadrant Phase-Mask Coronagraph. I. Principle," PASP 112, 1479-1486 (Nov. 2000).

[4] Serabyn, G., Mawet, D., and Burruss, R., "Imaging The Exoplanets In HR8799 With A Vector Vortex Coronagraph On The Palomar 1.5 M Diameter Well-corrected Subaperture," in [American Astronomical Society Meeting Abstracts \#215], Bulletin of the American Astronomical Society 42, \#377.06 (Jan. 2010).

[5] Mawet, D., Absil, O., Delacroix, C., Girard, J. H., Milli, J., O’Neal, J., Baudoz, P., Boccaletti, A., Bourget, P., Christiaens, V., Forsberg, P., Gonte, F., Habraken, S., Hanot, C., Karlsson, M., Kasper, M., Lizon, J.-L., Muzic, K., Olivier, R., Peña, E., Slusarenko, N., Tacconi-Garman, L. E., and Surdej, J., "L'-band AGPM vector vortex coronagraph's first light on VLT/NACO. Discovery of a late-type companion at two beamwidths from an F0V star," A\&A 552, L13 (Apr. 2013).

[6] Carlotti, A., "Apodized phase mask coronagraphs for arbitrary apertures," A\&A 551, A10 (Mar. 2013).

[7] Fourer, R., Gay, D., and Kernighan, B., "A modeling language for mathematical programming," Manage. Sci. 36, 519-554 (1990).

[8] Vanderbei, R., "LOQO: An interior point code for quadratic programming," Optim. Method. Softw. 12, 451-484 (1999).

[9] Gurobi Optimization, I., "Gurobi optimizer reference manual," (2013).

[10] Carlotti, A., Vanderbei, R., and Kasdin, N. J., "Optimal pupil apodizations of arbitrary apertures for high-contrast imaging," Optics Express 19, 26796 (Dec. 2011).

[11] Spergel, D. and Kasdin, J., "A Shaped Pupil Coronagraph: A Simpler Path towards TPF," in [American Astronomical Society Meeting Abstracts], Bulletin of the American Astronomical Society 33, 1431 (Dec. 2001).

[12] Vanderbei, R. J., Spergel, D. N., and Kasdin, N. J., "Circularly Symmetric Apodization via Star-shaped Masks," ApJ 599, 686-694 (Dec. 2003).

[13] Guyon, O., Pluzhnik, E. A., Kuchner, M. J., Collins, B., and Ridgway, S. T., "Theoretical Limits on Extrasolar Terrestrial Planet Detection with Coronagraphs," ApJS 167, 81-99 (Nov. 2006).

[14] Spergel, D., Gehrels, N., Breckinridge, J., Donahue, M., Dressler, A., Gaudi, B. S., Greene, T., Guyon, O., Hirata, C., Kalirai, J., Kasdin, N. J., Moos, W., Perlmutter, S., Postman, M., Rauscher, B., Rhodes, J., Wang, Y., Weinberg, D., Centrella, J., Traub, W., Baltay, C., Colbert, J., Bennett, D., Kiessling, A., Macintosh, B., Merten, J., Mortonson, M., Penny, M., Rozo, E., Savransky, D., Stapelfeldt, K., Zu, Y., Baker, C., Cheng, E., Content, D., Dooley, J., Foote, M., Goullioud, R., Grady, K., Jackson, C., Kruk, J., Levine, M., Melton, M., Peddie, C., Ruffa, J., and Shaklan, S., "WFIRST-2.4: What Every Astronomer Should Know," ArXiv e-prints (May 2013).

[15] Pueyo, L. and Norman, C., "High-contrast Imaging with an Arbitrary Aperture: Active Compensation of Aperture Discontinuities," ApJ 769, 102 (June 2013).

[16] Enya, K., Abe, L., Tanaka, S., Nakagawa, T., Haze, K., Sato, T., and Wakayama, T., "High contrast experiment of an AO-free coronagraph with a checkerboard pupil mask," A\&A 480, 899-903 (Mar. 2008). 